\title{
Shenfun: High performance spectral Galerkin computing platform
}

\section{Mikael Mortensen ${ }^{1}$}

1 University of Oslo, Department of Mathematics

DOI: $10.21105 /$ joss. 01071

\section{Software}

- Review ct

- Repository u

- Archive ${ }^{\top}$

Submitted: 07 November 2018

Published: 19 November 2018

\section{License}

Authors of papers retain copyright and release the work under a Creative Commons Attribution 4.0 International License (CC-BY).

\section{Summary}

Shenfun is an open-source computing platform for solving partial differential equations (PDEs) by the spectral Galerkin method. The user interface to shenfun is very similar to FEniCS (https://fenicsproject.org), but applications are limited to simple, yet multidimensional, tensor product grids.

Shenfun enables fast development of efficient and accurate PDE solvers (spectral order and accuracy), in the comfortable high-level Python language. The spectral accuracy is ensured by using high-order global orthogonal basis functions (Fourier, Legendre and Chebyshev), as opposed to finite element codes like FEniCS that are using low-order local basis functions. Efficiency is ensured through vectorization, parallelization (MPI) and by moving critical routines to Cython (https://cython.org).

With shenfun one can solve a wide range of PDEs, with the limitation that one dimension can be inhomogeneous (with Dirichlet/Neumann type of boundaries), whereas the remaining dimensions are required to be periodic. The periodic dimensions are discretized using Fourier exponentials as basis functions. For the inhomogeneous direction, we use combinations of Chebyshev or Legendre polynomials, as described by J. Shen (Shen, 1994, 1995).

The code is parallelized with MPI through the mpi4py-fft (https://bitbucket.org/mpi4py/ mpi4py-fft) package, and has been run for thousands of processors on various supercomputers. The parallelization is automated and highly configurable (slab, pencil), using a new algorithm (Dalcín, Mortensen, \& Keyes, 2018) for global redistribution of multidimensional arrays.

Shenfun is documented, with installation instructions and demo programs, on readthedocs (http://shenfun.readthedocs.org). Extended demonstration programs are included for, e.g., the Poisson, Klein-Gordon and Kuramato-Sivashinsky equations.

Shenfun has been designed as a low entry-level research tool for physicists (Mortensen, 2017a) in need of highly accurate numerical methods for high performance computing. The primary target for the development has been turbulence and transition to turbulence, where simulations are extremely sensitive to disturbances, and numerical diffusion or dispersion are unacceptable. Spectral methods are well known for their accuracy and efficiency, taking advantage of fast transform methods, like the Fast Fourier Transforms (FFTs). Combined with the Galerkin method and Shen's robust composite bases, this leads to well conditioned linear algebra systems and numerical schemes that are truly exceptional. Highly efficient direct solvers (Mortensen, 2017a) are provided for Poisson, Helmholtz and Biharmonic systems of equations, arising naturally with the current bases for a wide range of problems. 
In the spectralDNS repository (https://github.com/spectralDNS/spectralDNS) there are applications utilizing shenfun for forced isotropic turbulence (Navier-Stokes equations), turbulent channel flows (Mortensen, 2017b), Magnetohydrodynamics (MHD) and Rayleigh-Bénard flow. However, shenfun is by no means limited to the equations for fluid and plasma flows and it should be equally efficient for other scientific applications.

\section{Acknowledgements}

We acknowledge support from the 4DSpace Strategic Research Initiative at the University of Oslo

\section{References}

Dalcín, L., Mortensen, M., \& Keyes, D. E. (2018). Fast parallel multidimensional FFT using advanced MPI. CoRR, abs/1804.09536. Retrieved from http://arxiv.org/abs/1804. 09536

Mortensen, M. (2017a). Shenfun - automating the spectral galerkin method. In B. H. Skallerud \& H. I. Andersson (Eds.), MekIT'17 - ninth national conference on computational mechanics (pp. 273-298). International Center for Numerical Methods in Engineering (CIMNE).

Mortensen, M. (2017b). A spectral-galerkin turbulent channel flow solver for large-scale simulations. CoRR, abs/1701.03787. Retrieved from https://arxiv.org/abs/1701.03787

Shen, J. (1994). Efficient spectral-galerkin method i. Direct solvers of second- and fourthorder equations using legendre polynomials. SIAM Journal on Scientific Computing, 15(6), 1489-1505. doi:10.1137/0915089

Shen, J. (1995). Efficient spectral-galerkin method ii. Direct solvers of second- and fourthorder equations using chebyshev polynomials. SIAM Journal on Scientific Computing, 16(1), 74-87. doi:10.1137/0916006 